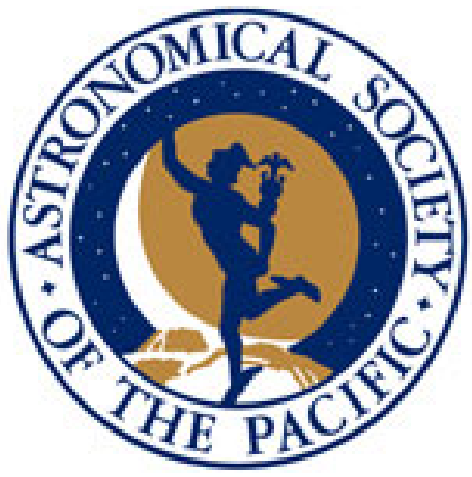

Airglow and Aurorae at Dome A, Antarctica

Author(s): Geoff Sims, Michael C. B. Ashley, Xiangqun Cui, Jon R. Everett, LongLong Feng, Xuefei Gong, Shane Hengst, Zhongwen Hu, Jon S. Lawrence, Daniel M. Luong-Van, Anna M. Moore, Reed Riddle, Zhaohui Shang, John W. V. Storey, Nick Tothill, Tony Travouillon, Lifan Wang, Huigen Yang, Ji Yang, Xu Zhou and Zhenxi Zhu

Reviewed work(s):

Source: Publications of the Astronomical Society of the Pacific, Vol. 124, No. 916 (June 2012), pp. 637-649

Published by: The University of Chicago Press on behalf of the Astronomical Society of the Pacific

Stable URL: http://www.jstor.org/stable/10.1086/666861

Accessed: 09/08/2012 00:08

Your use of the JSTOR archive indicates your acceptance of the Terms \& Conditions of Use, available at http://www.jstor.org/page/info/about/policies/terms.jsp

JSTOR is a not-for-profit service that helps scholars, researchers, and students discover, use, and build upon a wide range of content in a trusted digital archive. We use information technology and tools to increase productivity and facilitate new forms of scholarship. For more information about JSTOR, please contact support@jstor.org. 
Publications of the Astronomical Society of the Pacific, 124:637-649, 2012 June

(C) 2012. The Astronomical Society of the Pacific. All rights reserved. Printed in U.S.A.

\title{
Airglow and Aurorae at Dome A, Antarctica
}

\author{
Geoff Sims,${ }^{1}$ Michael C. B. Ashley, ${ }^{1}$ Xiangqun Cui, ${ }^{2}$ Jon R. Everett, ${ }^{1}$ Longlong Feng,,${ }^{3,4}$ Xuefei Gong, ${ }^{2,4}$ \\ Shane Hengst, ${ }^{1}$ Zhongwen Hu, ${ }^{2,4}$ Jon S. Lawrence, ${ }^{5,6}$ Daniel M. Luong-Van, ${ }^{1}$ Anna M. Moore, ${ }^{7}$ \\ Reed Riddle, ${ }^{7}$ Zhaohui Shang,,${ }^{4,8}$ John W. V. Storey, ${ }^{1}$ Nick Tothill, ${ }^{9}$ Tony Travouillon, ${ }^{7}$ \\ Lifan Wang, ${ }^{3,410}$ Huigen YAng, ${ }^{4,11}$ Ji YANG, ${ }^{3}$ XU Zhou, ${ }^{4,12}$ AND ZhenXI Zhu ${ }^{3,4}$ \\ Received 2012 April 26; accepted 2012 May 22; published 2012 June 15
}

\begin{abstract}
Despite the absence of artificial light pollution at Antarctic plateau sites such as Dome A, other factors such as airglow, aurorae, and extended periods of twilight have the potential to adversely affect optical observations. We present a statistical analysis of the airglow and aurorae at Dome A using spectroscopic data from Nigel, an optical/near-IR spectrometer operating in the 300-850 $\mathrm{nm}$ range. These data complement photometric images from Gattini, a wide-field $\left(90^{\circ}\right) \mathrm{CCD}$ camera with $B, V$, and $R$ filters, allowing the background sky brightness to be disentangled from the various airglow and auroral emission lines. The median auroral contribution to the $B, V$, and $R$ photometric bands is found to be $22.9,23.4$, and $23.0 \mathrm{mag} \mathrm{arcsec}^{-2}$, respectively. Auroral emissions most frequently occur between 10-23 hr local time, when up to 50\% of observations are above airglow-level intensities. While infrequent, the strongest emissions detected occurred in the hours just prior to magnetic midnight. We are also able to quantify the amount of annual dark time available as a function of wavelength, as well as in the standard $B V R$ photometric bands. On average, twilight ends when the Sun reaches a zenith distance of $102.6^{\circ}$.
\end{abstract}

Online material: color figures

\section{INTRODUCTION}

Located at the highest point on the Antarctic plateau, Dome A is considered one of the best sites for optical, infrared (IR), and submillimeter astronomy on Earth. Following the installation of the robotic Plateau Observatory (PLATO; Yang et al. 2009; Lawrence et al. 2009) in 2008 by the 25th Chinese National Antarctic Research Expedition, a number of predictions about the site quality have been confirmed: site testing results have demonstrated an atmospheric boundary layer confined very close to the ground (Bonner et al. 2010), exceptional stability and transparency in the terahertz-frequency regime (Yang et al.

\footnotetext{
${ }^{1}$ School of Physics, University of New South Wales, Sydney NSW 2052, Australia; g.sims@unsw.edu.au.

${ }^{2}$ Nanjing Institude of Astronomical Optics \& Technology, Nanjing 210042, China.

${ }^{3}$ Purple Mountain Observatory, Nanjing 210008, China.

${ }^{4}$ Chinese Center for Antarctic Astronomy, China.

${ }^{5}$ Department of Physics and Astronomy, Macquarie University, Sydney NSW 2109, Australia.

${ }^{6}$ Australian Astronomical Observatory, Sydney NSW 1710, Australia.

${ }^{7}$ Caltech Optical Observatories, Pasadena, CA 91125.

${ }^{8}$ Tianjin Normal University, Tianjin 300074, China.

${ }^{9}$ University of Western Sydney, Sydney NSW, Australia.

${ }^{10}$ Department of Physics and Astronomy, Texas A\&M University, College Station 77843.

${ }^{11}$ Polar Research Institute of China, Shanghai 200136, China.

${ }^{12}$ National Astronomical Observatories, Chinese Academy of Science, Beijing 100012, China.
}

2010), minimal cloud cover and a dark sky background in the $i$ band (Zou et al. 2010), and an exceedingly low atmospheric water vapor content (Sims et al. 2012). Among the site properties yet to be quantified at Dome A are the optical sky brightness contributions from airglow and aurorae; the latter have the potential to severely affect optical and near-IR astronomical observations. Numerous studies have speculated on their contributions (e.g., Dempsey et al. 2005; Kenyon \& Storey 2006; Saunders et al. 2009), but until now, no ground-based measurements from the Antarctic plateau have been reported in an astronomical context.

At any location, and in the absence of aurorae, airglow is the dominant component of optical and near-IR night sky brightness (Benn \& Ellison 1998). Airglow is caused by a chain of events beginning with the dissociation of molecules by daytime solar UV radiation, followed by chemical reactions that produce excited states that decay with the emission of photons. Spectrally, airglow consists of a weak continuum in the blue (the $\mathrm{O}_{2}$ Herzberg bands) and in the green (from $\mathrm{NO}_{2}$ emissions), as well as stronger emissions predominantly from neutral atomic oxygen, [O I] $(557.7,630.0$, and $636.4 \mathrm{~nm})$, and hydroxyl radicals (the $\mathrm{OH}$ Meinel band; from $\sim 600-4500 \mathrm{~nm}$ ).

Occurring at similar heights and also being similarly emitted by excited atoms and molecules, the aurora is driven by a completely different mechanism: collisions with energetic particles (mainly electrons) originating from the solar wind, which have been directed along the Earth's magnetic field lines. As with the 
airglow, these collisions give rise to a host of emission lines. The blue region ( $U$ and $B$ bands) is dominated by the $\mathrm{N}_{2}$ second positive (2P) and $\mathrm{N}_{2}^{+}$first negative $(1 \mathrm{~N})$ bands, while in the green region ( $V$ band), the strongest emission is that of atomic oxygen $(557.7 \mathrm{~nm})$. Redward of $650 \mathrm{~nm}$, the $R$ and $I$ bands contain a quasi-continuum contribution predominantly from the $\mathrm{N}_{2}$ first positive (1P), $\mathrm{N}_{2}^{+}$Meinel (M), and $\mathrm{O}_{2}$ atmospheric (ATM) bands (Gattinger \& Jones 1974; Jones \& Gattinger 1975).

A comparison of a typical airglow and auroral spectrum is presented in Figure 1. Of particular interest is the [O I] $557.7 \mathrm{~nm}$ emission, which dominates the $V$ band in both phenomena. Indeed, the constant presence of this line prompted the use of terminology such as the permanent (Yntema 1909) or nonpolar (Rayleigh 1924) aurora for what is now commonly known as airglow. Accordingly, an auroral event cannot be confirmed based on the existence of this line alone. While the forbidden transition that emits the $557.7 \mathrm{~nm}$ photon is the same in both cases (see eq. [4]), the production of excited atomic oxygen, $\mathrm{O}\left({ }^{1} \mathrm{~S}\right)$, that precedes the emission is different. During the aurora, excitation to $\mathrm{O}\left({ }^{1} \mathrm{~S}\right)$ occurs directly via fast collisions with energetic particles (Chamberlain 1961).

In contrast, and as explained, e.g., in Shepherd et al. (1997), the [O I] $557.7 \mathrm{~nm}$ airglow emission begins with the initial dissociation of $\mathrm{O}_{2}$ molecules by solar $\mathrm{UV}$ radiation. With long lifetimes in the upper atmosphere (hours to days), the dissociated
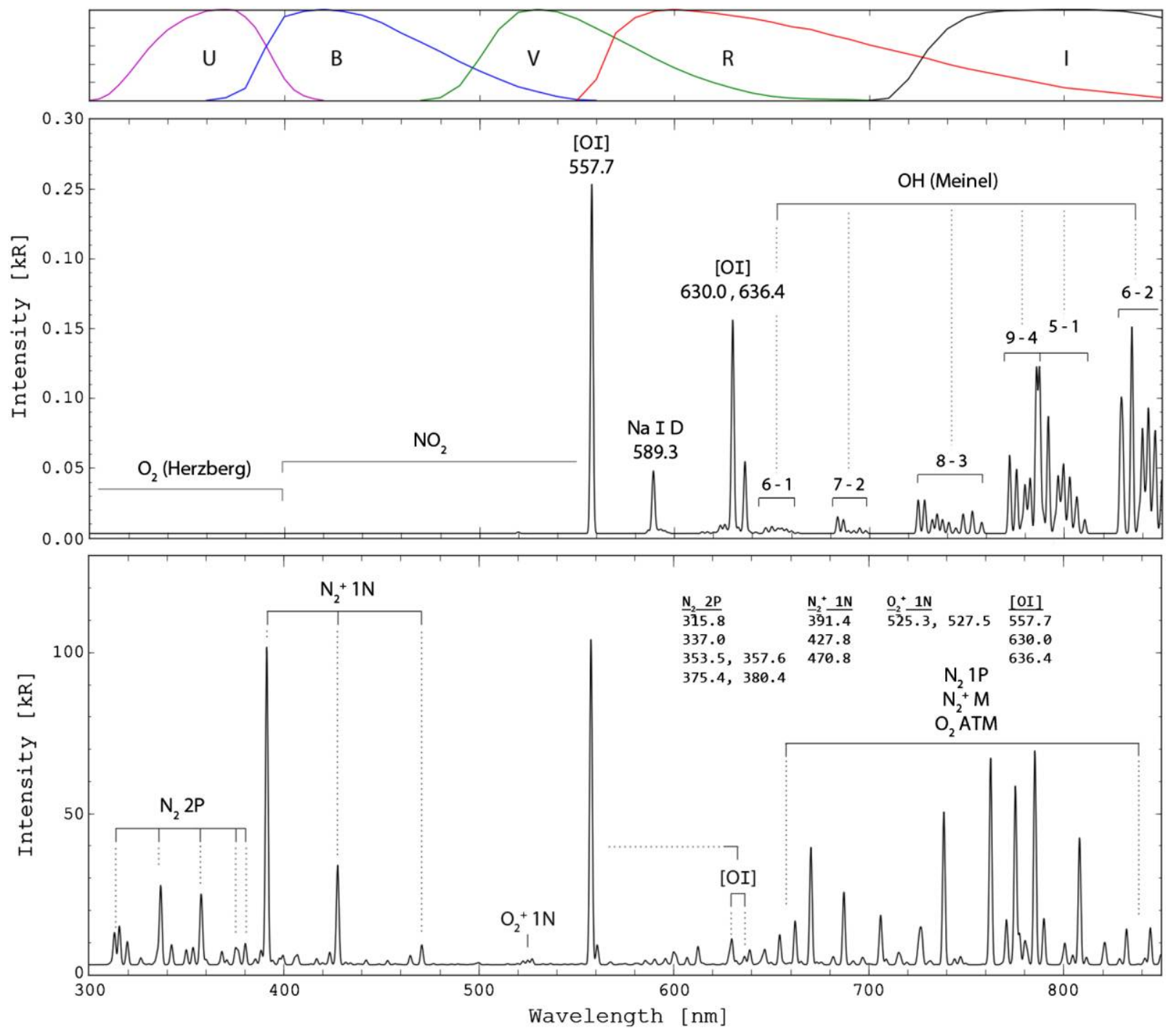

FIG. 1.-Synthetic airglow (middle) and auroral (bottom) spectrum, displayed at $1.5 \mathrm{~nm}$ resolution, showing typical intensities (in kilorayleighs) of dominant emissions in the 300-850 nm range. Wavelength identifications are in nanometers. Airglow emissions and intensities are from Meinel (1950a, 1950b) and Kvifte (1959). Auroral emissions and intensities are from Gattinger \& Jones (1974) and Jones \& Gattinger (1975) and represent an IBC III auroral event (normalized to an [O I] $557.7 \mathrm{~nm}$ intensity of $100 \mathrm{kR}$ ). Note the different scales used on the $y$-axes; the [O I] $557.7 \mathrm{~nm}$ emission is $\sim 400$ times greater in an IBC III aurora than it is in the typical airglow. Curves on the upper plot show the normalized $U B V R I$ photometric passbands. See the electronic edition of the PASP for a color version of this figure. 


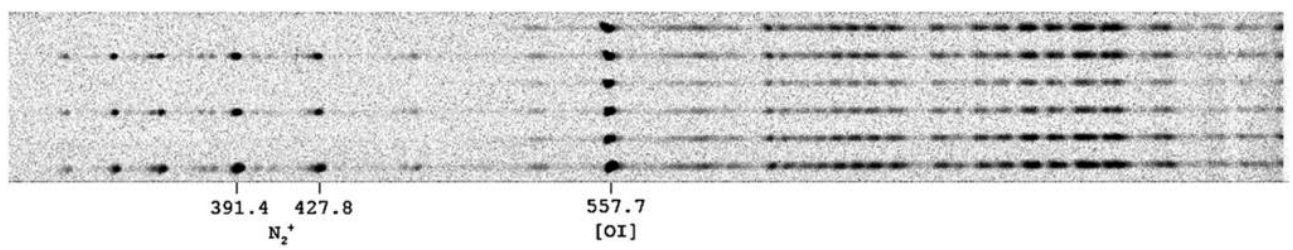

FIG. 2.-Raw Nigel image showing a bright aurora, taken on 2009 June 25 at 22:50 UT. The brightest emission lines are labeled (units are in nanometers). The six spectra represent blue and red optimized pairs from three directions on the sky.

oxygen atoms can be transported globally before recombination, explaining why the airglow is present at high latitudes even during the long polar winters. A two-step energy transfer process ensues, first involving a third molecule designated as $\mathrm{M}$ (usually $\mathrm{N}_{2}$ ), to produce an excited oxygen molecule, $\mathrm{O}_{2}^{*}$. This excited molecule subsequently transfers its energy to $\mathrm{O}$, leading to the excited state, $\mathrm{O}\left({ }^{1} \mathrm{~S}\right)$, and the forbidden 557.7 emission. The entire process is presented below:

$$
\begin{gathered}
\mathrm{O}_{2}+h \nu(\text { solar UV }) \rightarrow \mathrm{O}+\mathrm{O} \\
\mathrm{O}+\mathrm{O}+\mathrm{M} \rightarrow \mathrm{O}_{2}^{*}+\mathrm{M} \\
\mathrm{O}_{2}^{*}+\mathrm{O} \rightarrow \mathrm{O}_{2}+\mathrm{O}\left({ }^{1} \mathrm{~S}\right) \\
\mathrm{O}\left({ }^{1} \mathrm{~S}\right) \rightarrow \mathrm{O}\left({ }^{1} \mathrm{D}\right)+h \nu(557.7 \mathrm{~nm})
\end{gathered}
$$

Airglow emissions usually have a relatively small range of intensities, compared with auroral emissions that can range over factors of 1000 or more. Typical intensities, in rayleighs, ${ }^{13}$ were described by Chamberlain (1961): $250 \mathrm{R}$ for airglow; $1 \mathrm{kR}$ for an aurora comparable with the brightness of the Milky Way; $10 \mathrm{kR}$ for an aurora equivalent to the brightness of thin moonlit cirrus cloud; $100 \mathrm{kR}$ if equivalent to the brightness of moonlit cumulus clouds; and $1000 \mathrm{kR}$ if providing total illumination (at the ground) equivalent to full moonlight. These four somewhat broad "classes" of aurorae are designated by international brightness coefficients (IBCs) of I, II, III, and IV, respectively.

In this article, we briefly review the variability of airglow and aurorae, and we use spectroscopic observations to examine their contribution to sky brightness at Dome A in the $B V R$ photometric bands, as well as the characteristics of their nocturnal fluctuations. We are also able to investigate the amount of annual dark time as a function of wavelength - an important consideration given the extended periods of twilight that occur at polar latitudes.

\footnotetext{
${ }^{13} 1 \mathrm{R} \equiv 10^{6} / 4 \pi$ phot s$^{-1} \mathrm{~cm}^{-2} \mathrm{sr}^{-1} \equiv 1.24 \times 10^{7} \lambda(\mathrm{m}) \mu \mathrm{J} \operatorname{arcsec}^{-2}$.
}

\section{REVIEW OF AIRGLOW AND AURORAE VARIABILITY}

Although the purpose of this article is not to account for observed variations in airglow and aurorae, it is useful to review the spatiotemporal fluctuations of both phenomena to provide some context for the observations.

Airglow varies on both short and long timescales: atmospheric gravity waves are responsible for fluctuations on timescales of minutes (e.g., Li et al. 2011); solar tides produce diurnal (e.g., Brenton \& Silverman 1970; Fukuyama 1976) and semiannual (e.g., Deutsch \& Hernandez 2003) oscillations; and the solar cycle causes decade-scale variations, with sky brightness typically being 0.4 mag brighter during solar maximum (Krisciunas 1997; Patat 2008), with the $10.7 \mathrm{~cm}$ solar radio flux commonly used to measure the level of solar activity (e.g., Walker 1988). Temporal fluctuations also display a complex dependence on geographic latitude (e.g., Forsyth \& Wraight 1987). Nightly variations of the overall sky brightness are usually low (<0.1 mag; Benn \& Ellison 1998) but can occasionally be much higher (up to 0.7 mag; Patat 2008).

Unlike airglow, which occurs globally, aurorae are generally confined to a region known as the auroral oval, an oval-shaped annulus centered on the geomagnetic poles. It has long been recognized that this region varies in size and shape with both geomagnetic activity (Feldstein \& Starkov 1967) and southward component of the interplanetary magnetic field (IMF; Holzworth \& Meng 1975). Simmons (1998) has reviewed the various types of aurora and their dependence on geomagnetic latitude, magnetic local time (MLT), ${ }^{14}$ and the mechanism of production. In the vicinity of the auroral oval, magnetic substorms occur frequently with a maximum just prior to magnetic midnight (Simmons 1998; Akasofu 2010). While auroral activity at locations between the equator and the auroral oval is correlated with solar cycle, the situation inside the auroral oval (or polar cap) is more complicated. Here, even in the strongest magnetic storms, visual aurorae are often not observed (Sandford 1961). Two broad classes of aurorae occur in these

\footnotetext{
${ }^{14}$ MLT is defined as the hour angle between the geomagnetic meridian through the site and the direction away from the Sun (see review by von Biel 1990).
} 
regions: discrete (having structure) and diffuse (widespread; filling most of the sky).

Discrete polar cap aurorae display two intensity maxima: one at 22-24 hr MLT with an occurrence frequency proportional to magnetic activity and another at $09-10 \mathrm{hr}$ MLT, which is anticorrelated with magnetic activity (Hartz \& Brice 1967; Sandford 1968). Two subsets of discrete polar aurora include polar cap arcs and polar cap patches. Polar cap arcs occur during quiet magnetic activity and northward IMF (Zhu et al. 1997) and are 4 times more common in magnetic dawn than dusk (Hosokawa et al. 2011), while polar cap patches exhibit a maximum at $23 \mathrm{hr}$ MLT with intensities several times that of airglow (typically 1 kR; McEwen \& Harrington 1991; Moen et al. 2007).

Diffuse aurora are usually subvisual and include the following subclasses: polar glow and polar rain. The polar glow is caused by bombardment by high-energy protons and alpha particles following large solar flares, and it may last for up to 3-4 days (Sandford 1962), which causes major enhancements to the $\mathrm{N}_{2} 1 \mathrm{~N}, 1 \mathrm{P}$, and 2P bands (Simmons \& Henriksen 1995). The intensity maxima of polar glow occurs at 18-24 hr MLT (Simmons 1998). Polar rain, caused by the constant bombardment of lowerenergy particles from the solar wind (Winningham \& Heikkila 1974), is dependent on solar cycle (Gussenhoven \& Madden 1990) and IMF (Newell et al. 2009). The energy flux of the lower-energy particles is generally weak, with stronger events occurring a few times annually (Newell et al. 2009).

As a general remark, Sandford (1964) reports that $75-85 \%$ of emissions at high magnetic latitudes are attributable to nonvisual aurorae and that even during quiet magnetic conditions, the $\mathrm{N}_{2}^{+} 391.4 \mathrm{~nm}$ line shows measurable ( $>100 \mathrm{R}$ ) intensities. As an example of subvisual auroral intensities (and excluding the airglow component), Vorobjev et al. (2008) have reported isolated observations of $100-300 \mathrm{R}$ and $200-500 \mathrm{R}$ in the [O I] 557.7 and $630.0 \mathrm{~nm}$ lines, respectively.

\section{OBSERVATIONS AND DATA REDUCTION}

The data sets used in this article were acquired during 2009 using two instruments at Dome A. The first and primary instrument is a spectrometer named Nigel operating in the optical to near-IR (300-850 nm) spectral regime (Sims et al. 2010). Nigel continuously records spectra of the daytime, twilight, and night sky using optical fibers with no additional optics. The field of view (FOV) is therefore determined solely by the numerical aperture (NA) of the fibers; in our case the NA is 0.22, which gives rise to a $25^{\circ}$ diameter FOV. At $500 \mathrm{~nm}$ the achieved resolution is approximately $3 \mathrm{~nm}$ FWHM with $0.6 \mathrm{~nm}$ pixel $^{-1}$. Although Nigel collects spectra from three fixed directions on the sky, for this analysis we consider only data obtained from the zenith direction. Exposure times are set automatically depending on the flux and vary from $1 \mathrm{~s}$ when the Sun is above the horizon to $512 \mathrm{~s}$ during the dark winter nights.

The second instrument, used in this context primarily as a calibrator for Nigel, is a CCD camera (Apogee Alta U4000)

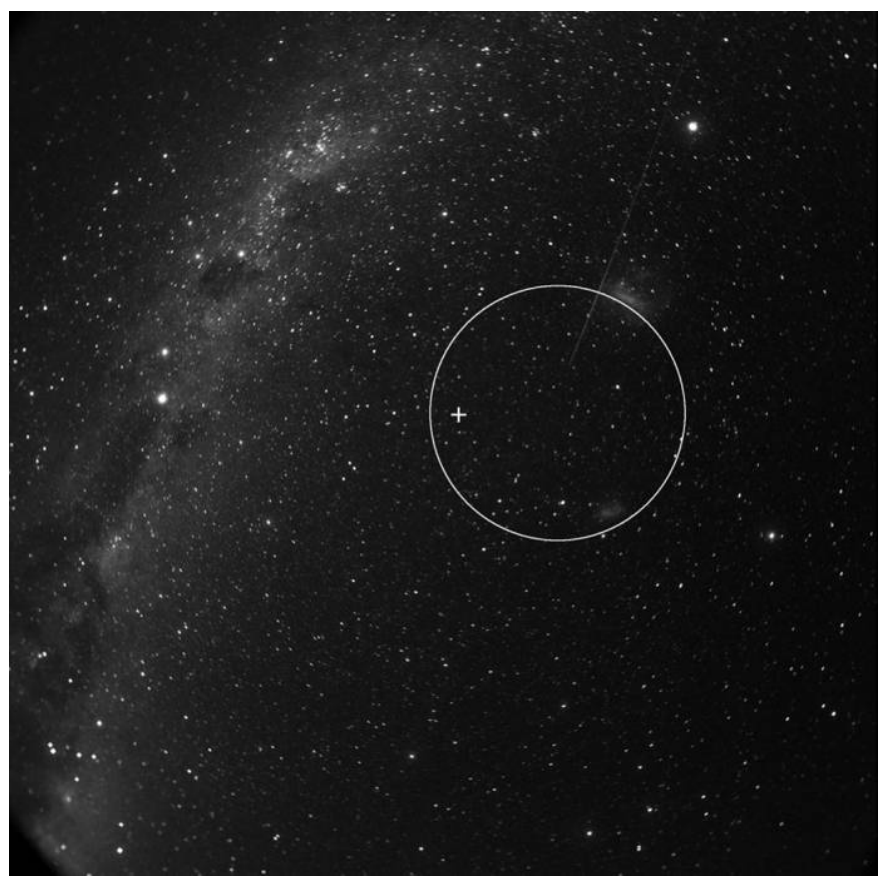

FIG. 3.-Raw Gattini image (100 s exposure in $V$, taken on 2009 July 20 at 01:55 UT) showing the approximate area of Nigel's zenith field of view. The cross marks the position of the south celestial pole. The streak through the image is a satellite trail. Other distinguishing features include the Large and Small Magellanic Clouds (right-hand edge of the Nigel field); the Southern Cross and pointers (left-hand side above center); and the bright star Canopus (upper right)

coupled to a $90^{\circ}$ fisheye lens, named Gattini (Moore et al. 2010). The camera has a $2048 \times 2048$ array of $7.4 \mu \mathrm{m}^{2}$ pixels. Included with the camera is a set of Bessell $B, V$, and $R$ photometric filters, as well as a long-pass red filter to detect $\mathrm{OH}$ emissions. Gattini also contains a "dark" filter, which enables bias and dark frames to be taken despite the use of a shutterless interline CCD. The image scale of Gattini in the zenith direction averages 148" per pixel over the equivalent Nigel FOV. Exposure times on Gattini are set in a similar fashion to Nigel, but are fixed during winter when consecutive exposures of $30 \mathrm{~s}$ and $100 \mathrm{~s}$ are taken for each filter.

The Nigel reduction pipeline is as follows (note that because of shutterless operation of the CCD camera, no bias or dark frames are taken). First, the images are bias-subtracted using the median value from a four-column overscan region to estimate

TABLE 1

Photometric Zero Points for Various Passbands

\begin{tabular}{llcc}
\hline \hline & Filter & No. stars & $m_{0}$ \\
\hline$B$ & $\ldots \ldots \ldots \ldots \ldots$ & 15 & $11.0 \pm 0.1$ \\
$V$ & $\ldots \ldots \ldots \ldots \ldots$ & 13 & $11.3 \pm 0.1$ \\
$R$ & $\ldots \ldots \ldots \ldots \ldots$ & 9 & $10.9 \pm 0.1$ \\
\hline
\end{tabular}

Note.-The error listed is $1 \sigma$ 


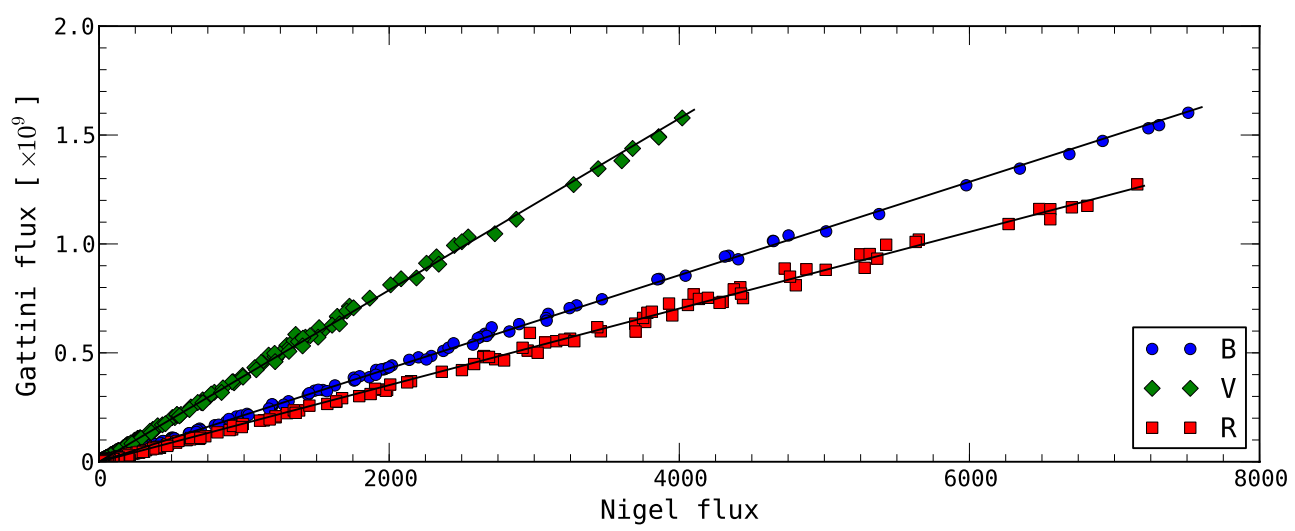

FIG. 4.-Nigel-Gattini relationship for the $B, V$, and $R$ passbands. The data shown span both twilight and night time observations (solar zenith distance ranges of 95.5-115. $0^{\circ}$ ). See the electronic edition of the PASP for a color version of this figure.

the bias contribution. Following bias subtraction, an empirical relationship between dark current and CCD temperature is created by examining nonspectra pixels from images at various temperatures; this dark current is subsequently subtracted from each pixel. Next, a cosmic-ray removal algorithm (Pych 2004) is employed to remove hot pixels. The parameters of the Pych algorithm were carefully chosen to make sure no emission lines were erroneously detected as hot pixels or cosmic rays. The spectra were then extracted using optimal spectral extraction (a Gaussian mask in the cross-spectral direction) and normalized to the exposure time. A sample raw image, prior to spectral extraction, is shown in Figure 2. The emission lines have low signal-to-noise ratios $(\mathrm{S} / \mathrm{N})$, limited by quantization noise. A second, more aggressive, cosmic-ray removal algorithm (van Dokkum 2001) was then employed to remove cosmic rays.
Finally, the spectra were corrected for the expected instrumental response of the system (Sims et al. 2010). A small fraction $(0.5 \%)$ of images were excluded as a result of glitches in the algorithm that selected exposure times. The total number of images available for analysis is 54,936, of which $20 \%$ were taken during times when the Sun was more than $15^{\circ}$ below the horizon.

A more basic reduction process is used with the Gattini images, consisting solely of bias subtraction and exposure time normaliziation. As with Nigel, a single bias value is determined for each image using the overscan region. A small exposure time offset $(+0.0025 \mathrm{~s})$ is added prior to exposure time normalization, as a suspected electronic delay causes the exposure time to be slightly longer than what is recorded in the FITS header. A number of images taken early in the year, known to be affected
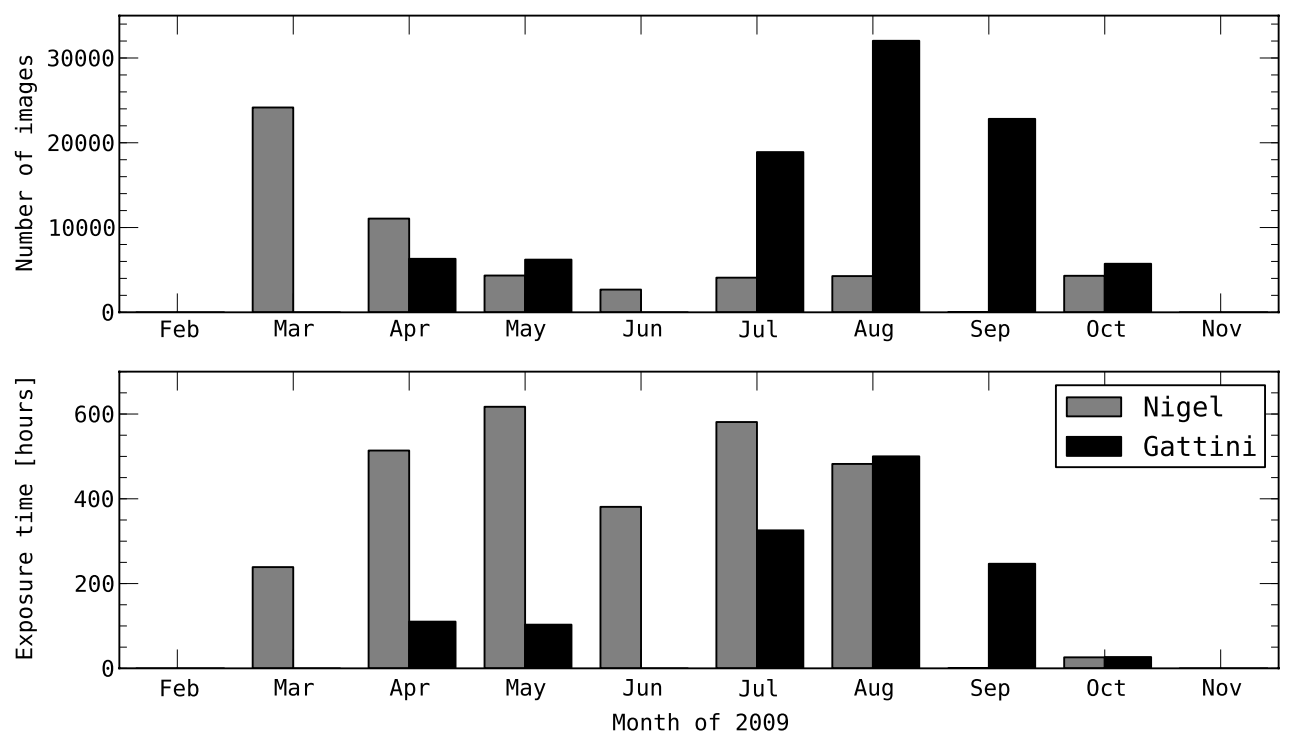

FIG. 5.-Overview of Nigel and Gattini images during 2009, showing the total number of images (top) and cumulative exposure time (bottom). The Gattini numbers include ice-affected exposures and calibration frames. Gaps in the data represent times when either the instruments or computers were temporarily offline. 

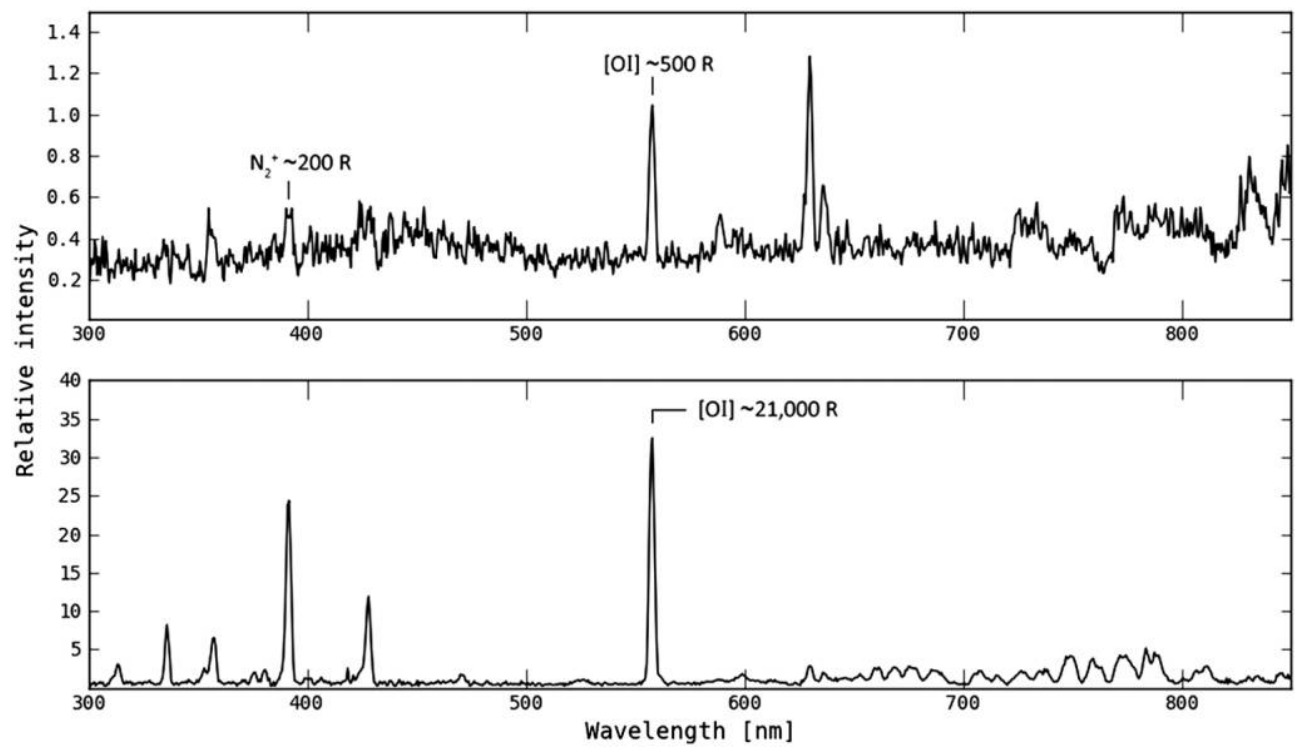

FIG. 6. - Sample Nigel data showing an airglow spectrum (top) and a bright auroral event (bottom). The airglow spectrum is a median combine of over 7000 observations to increase the $\mathrm{S} / \mathrm{N}$.

by ice accumulation on the lens, were excluded from further analysis. The total number of Gattini images available for analysis is 74,471 . Figure 3 shows a raw Gattini image with the expected Nigel FOV overlaid.

\section{PHOTOMETRIC CALIBRATION AND ERRORS}

To achieve photometric calibration on the Gattini images, aperture photometry was performed on a number of stars using IRAF. With stars brighter than magnitude 2 showing signs of saturation, the magnitude, $m$, of stars selected for photometry was confined to $2<m<5$. In this preliminary analysis, which is done purely to provide a calibration for Nigel, no air-mass or flat-field corrections were applied (this will be followed up by a more thorough analysis in a subsequent article; Yang et al. 2012, in preparation). Instrumental magnitudes, $m_{i}$, were calculated for each star on each image, according to

$$
m_{i}=25-2.5 \log _{10}\left(\frac{f_{\text {star }}-f_{\text {sky }}}{t_{\text {exp }}}\right),
$$

where $f_{\text {star }}$ and $f_{\text {sky }}$ are the star and sky fluxes, respectively, and $t_{\exp }$ is the exposure time. With typically 2000 observations in which each star was accurately measured, the stars' overall instrumental magnitudes were calculated by choosing the brightest 10th percentile value-intentionally biased toward bright observations to give a better estimate for the star's brightness in the absence of cloud, aurora, etc. The photometric zero point, $m_{0}$, was determined by comparing each star with its catalog magnitude, with the final estimate being the mean of all results. Table 1 gives the values of and errors in $m_{0}$ for each passband.
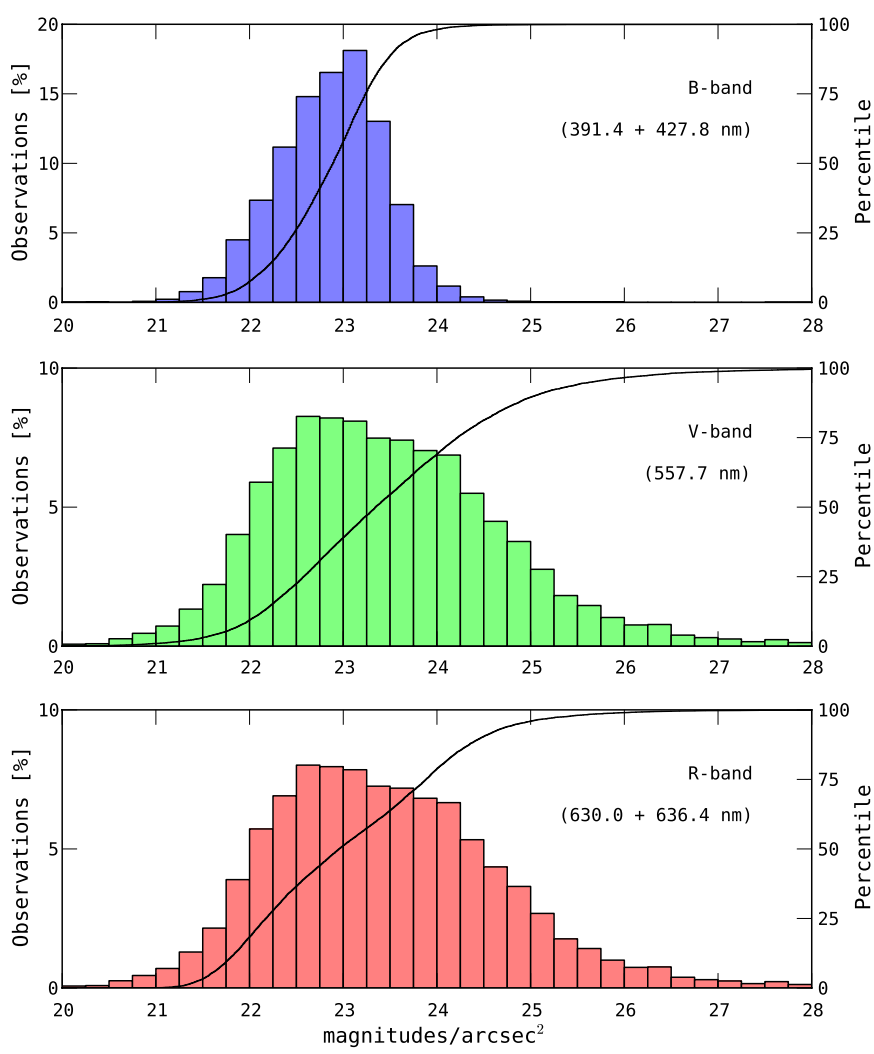

FIG. 7.-Probability histograms showing the sky brightness contributions from the brightest emission lines in each photometric band. The $B$ band includes $\mathrm{N}_{2}^{+} 391.4$ and $427.8 \mathrm{~nm}$; the $V$ band consists of [O I] $557.7 \mathrm{~nm}$; and the $R$ band includes [O I] 630.0 and $636.4 \mathrm{~nm}$. See the electronic edition of the $P A S P$ for a color version of this figure. 
TABLE 2

Quartile Values of Dominant Emission-Line Contributions to SKy BRIGHTNESS IN $B V R$ PHOTOMETRIC BANDS

\begin{tabular}{ccccc}
\hline \hline \multicolumn{2}{c}{ Filter } & Best 25\% & Median & Best 75\% \\
\hline$B$ & $\ldots \ldots \ldots$ & 23.2 & 22.9 & 22.5 \\
$V$ & $\ldots \ldots \ldots$ & 24.2 & 23.4 & 22.6 \\
$R$ & $\ldots \ldots \ldots$ & 23.9 & 23.0 & 22.2 \\
\hline
\end{tabular}

NoTE.-Units are mag $\operatorname{arcsec}^{-2}$.

With the photometric zero point determined, the surface brightness, $b$, in the $B, V$, and $R$ bands can be determined for any arbitrary part of a Gattini image using

$$
b=m_{0}-2.5 \log _{10}\left(\frac{f-\left(N \times f_{\text {bias }}\right)}{N p^{2} t_{\exp }}\right),
$$

where $f$ is the total flux in the region under consideration, $N$ is the area of the region in pixels, $f_{\text {bias }}$ is the bias per pixel, and $p$ is the image scale in arcsec pixel ${ }^{-1}$.

In order to apply the photometric calibration to the Nigel spectra, each Nigel spectrum was multiplied with $B, V$, and $R$ filter profiles and integrated to simulate the various passbands. These were compared with the flux obtained from contemporaneous Gattini images over the expected Nigel FOV. The Nigel fluxes for each filter had been interpolated to match the epoch of the Gattini images. Figure 4 shows the results for each filter. The correlation coefficients were all greater than 0.99. A comparison of the temporal distribution and total integration times of Nigel and Gattini is given in Figure 5.

\section{AIRGLOW AND AURORAL CONTRIBUTIONS TO SKY BRIGHTNESS}

To investigate the intensities of the airglow and aurorae during "night" time, a subset of the Nigel data in which the
Sun was below $-13^{\circ}$ was selected for use. Each of these 12,786 spectra were multiplied with $B, V$, and $R$ filter profiles, and the intensities of some dominant emissions were then measured: $\mathrm{N}_{2}^{+}$(391.4 and $427.8 \mathrm{~nm}$ ) and [O I] (557.7, 630.0, and $636.4 \mathrm{~nm}$ ). The uncalibrated intensities were converted into mag $\operatorname{arcsec}^{-2}$ (see $\S 4$ ). A small number of lines ( $0.5 \%$ of 391.4 and $427.8 \mathrm{~nm} ; 4 \%$ of $557.7 \mathrm{~nm}$; and $3.5 \%$ of 630.0 and $636.4 \mathrm{~nm}$ ) were unable to be measured, most likely as a result of being too weak.

Two sample spectra, representing a typical airglow spectrum and strong auroral display, are shown in Figure 6. Difficulties in splicing together the calibrated $B V R$ portions of the spectra prevent an absolute scale being used over the entire wavelength interval. The arbitrary scale is normalized to the [O I] $557.7 \mathrm{~nm}$ line in the airglow spectrum in both cases; hence, the relative intensity between the plots is correct. Most of the features identified in the synthetic spectra (Fig. 1) are visible and are in good agreement with typical predicted intensity ratios, although the pseudocontinuum redward of $600 \mathrm{~nm}$ in the auroral spectrum appears significantly weaker than expected. The intensity of the [O I] $557.7 \mathrm{~nm}$ line in the auroral spectrum is approximately $21 \mathrm{kR}$.

The airglow spectrum, which was produced by finding the median value of each pixel from more than 7000 dark sky observations, contains some surprising features: the apparent presence of $\mathrm{N}_{2} 2 \mathrm{P}$ (353.6 and $357.6 \mathrm{~nm}$ ) and $\mathrm{N}_{2}^{+} 1 \mathrm{~N}$ (391.4, 423.7, $427.8 \mathrm{~nm}$ ) emissions. These features are generally reported as only appearing in the airglow at extremely low intensities (e.g., less than 1 R; Broadfoot \& Kendall 1968; Meriwether \& Walker 1980), if at all. The $1 \mathrm{~N}$ emissions can, however, reach $\sim 50 \mathrm{R}$ during lightning strokes (Moore 1963; Patat 2008). The median value of the $\mathrm{N}_{2}^{+} 391.4 \mathrm{~nm}$ emission in our observations is $\sim 200 \mathrm{R}$.

To quantify the contribution of the major emission lines to sky brightness in the $B V R$ bands, probability histograms were produced and are shown in Figure 7. The corresponding

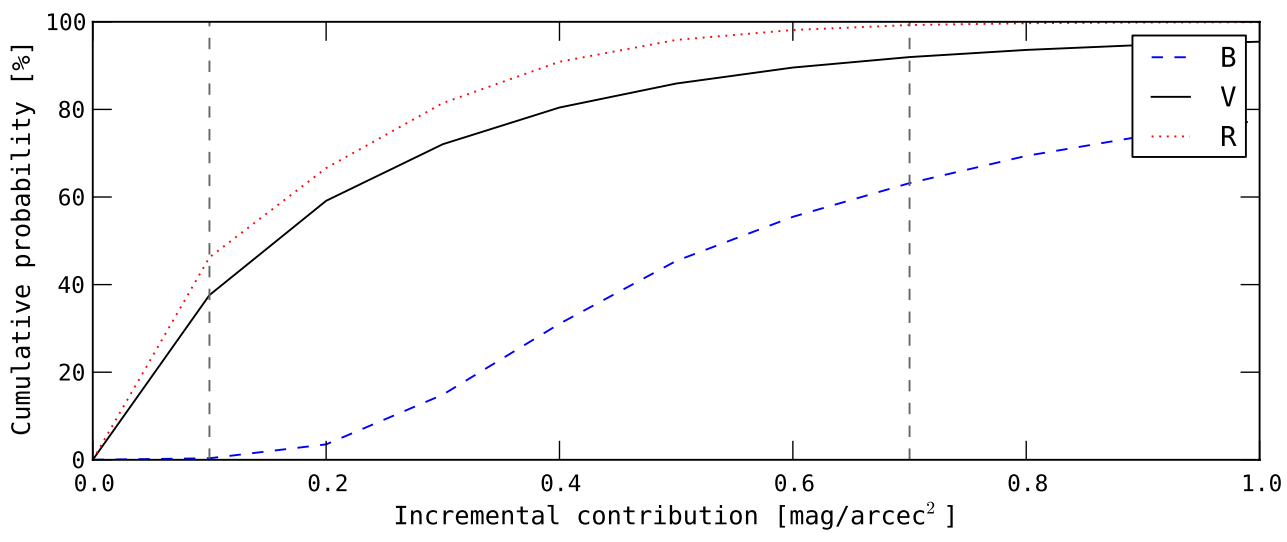

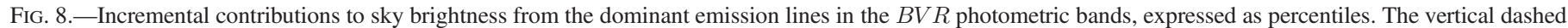

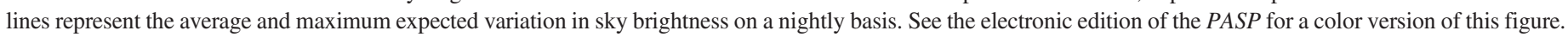



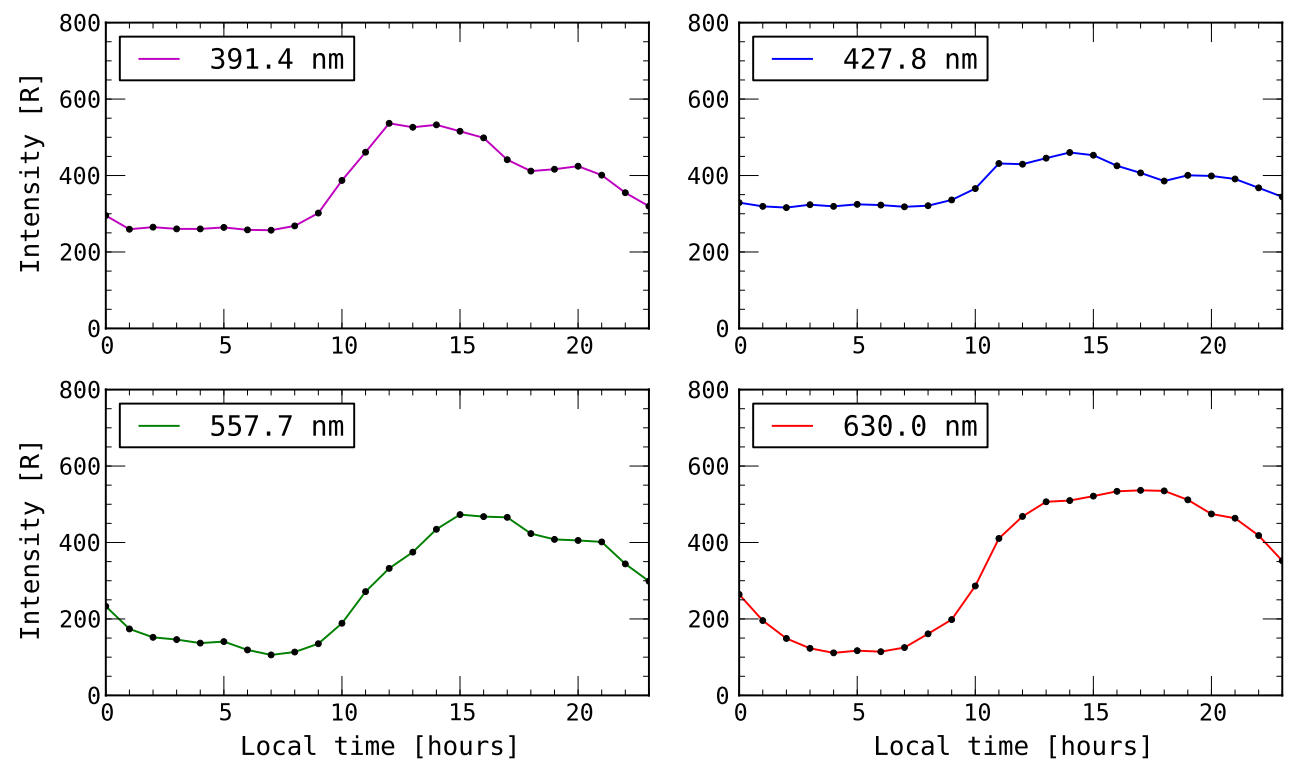

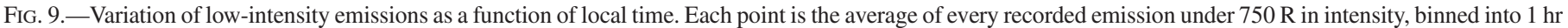
intervals. A broad minimum is visible after local midnight. See the electronic edition of the PASP for a color version of this figure.

tabulated quartile values are shown in Table 2. The median contribution intensities are in good agreement with the modeling performed in Saunders et al. (2009), where an average of $23.0 \mathrm{mag} \operatorname{arcsec}^{-2}$ is quoted for the $B$ and $V$ bands. As a comparison, the zenith zodiacal light contribution at a midlatitude site such as Mauna Kea ranges from 23.1-22.1 mag $\operatorname{arcsec}^{-2}$, whereas at Dome A it is always darker than $23.1 \mathrm{mag} \mathrm{arcsec}^{-2}$ (Kenyon \& Storey 2006). In this respect, the zenith sky bright-
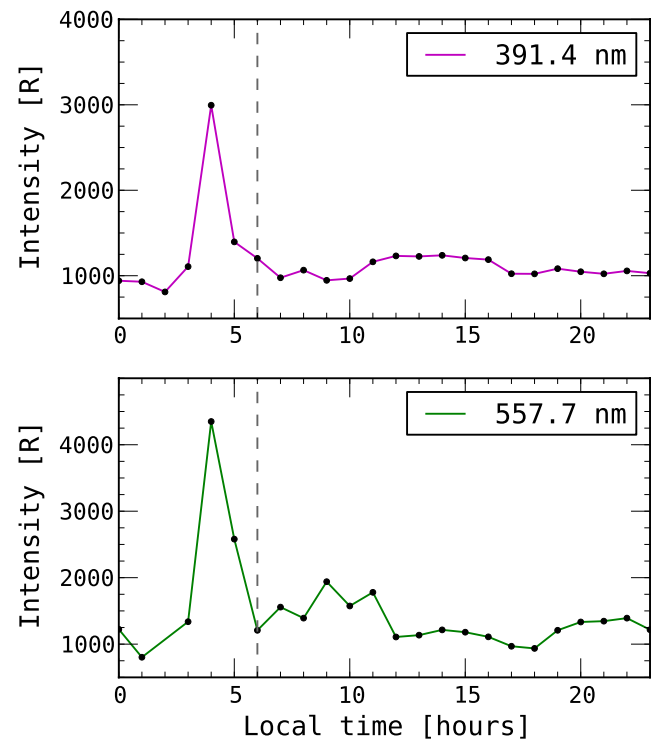

ness contribution from auroral emissions at Dome A is significantly less than the zodiacal light at midlatitude sites.

Using preliminary estimates for the sky brightness background obtained with Gattini, the incremental contributions to sky brightness were calculated, with the percentiles, shown in Figure 8, providing a useful metric for determining the impact of such emissions. In the $V$ and $R$ bands, the contribution is less than 0.2 mag more than $50 \%$ of the time and less than $0.5 \mathrm{mag}$
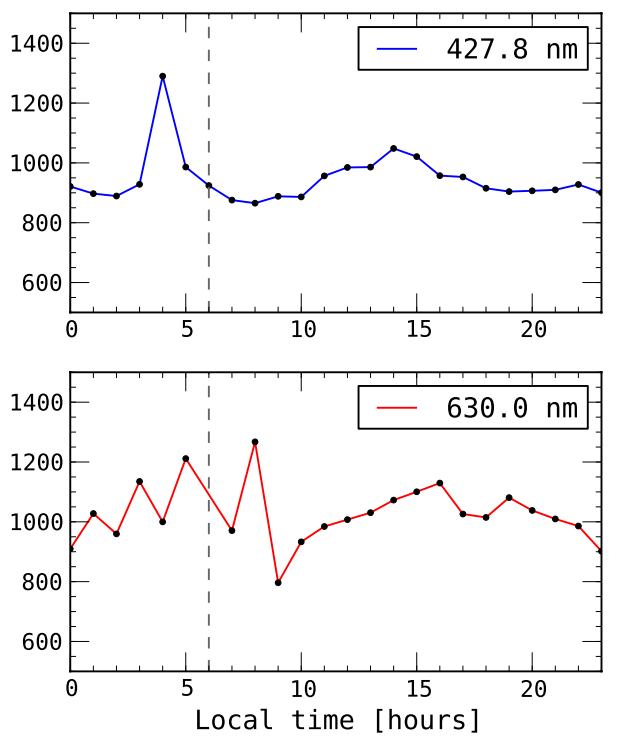

FIG. 10.-Same as Fig. 9 but for high intensities. The dashed vertical line represents magnetic midnight. A strong maximum exists for all lines (except 630.0 nm) around $4 \mathrm{hr}$ LT, shortly before magnetic midnight. A number of bins for particular lines did not contain any emissions above the specified threshold. See the electronic edition of the PASP for a color version of this figure. 

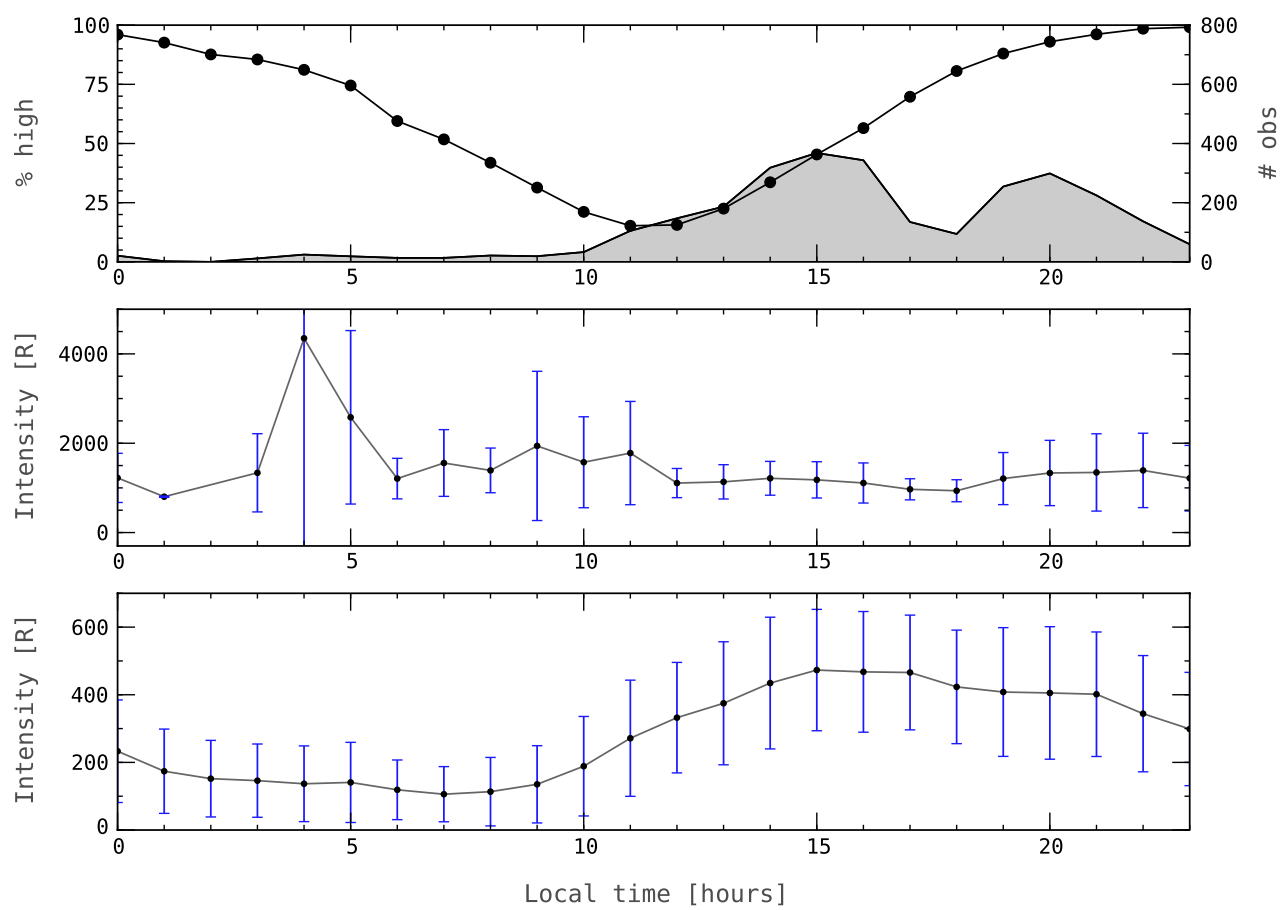

FIG. 11.-Top: Number of observations (line) and percentage with high intensities (shaded). Middle and Bottom: Variability of the high and low [O I] $557.7 \mathrm{~nm}$ emissions, respectively, from Figs. 9 and 10, but with error bars showing $\pm 1 \sigma$. See the electronic edition of the PASP for a color version of this figure.

more than $85 \%$ of the time. The contributions in the $B$ band are somewhat higher.

To examine the variability of the emissions, their contributions, in mag $\operatorname{arcsec}^{-2}$, were converted into rayleighs (see, e.g., Bessell 1979; Benn \& Ellison 1998; Dempsey et al. 2005), the most commonly used unit when studying the airglow and aurorae. Our photometric accuracy of \pm 0.1 mag corresponds to $\pm 9 \%$ in linear units such as rayleighs. The intensities were binned into $1 \mathrm{hr}$ bins of Local Time (LT).

In order to explore the temporal fluctuations of the airglow and auroral emissions, we need some way to characterize each observation as being predominantly resulting from either airglow or an aurora. Using the relative line intensities is difficult, because of the near-constant presence of low-intensity $\mathrm{N}_{2}^{+}$

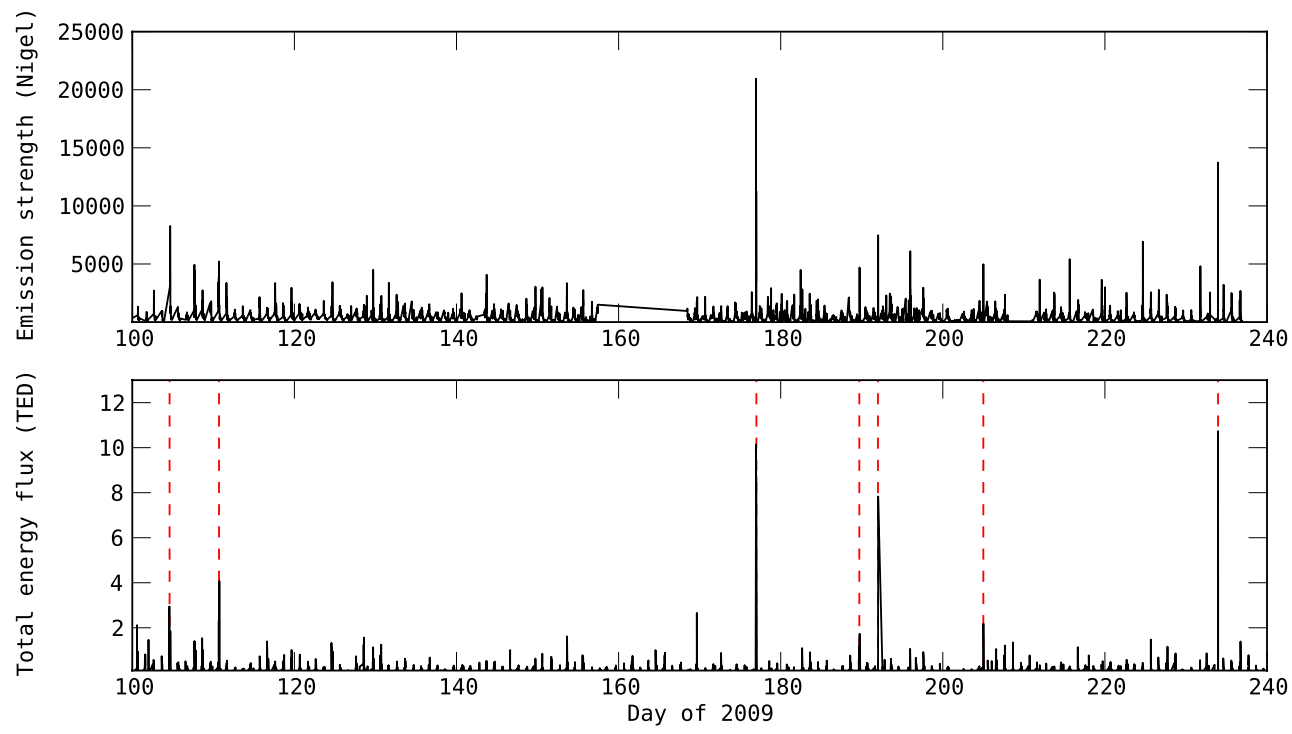

FIG. 12.-Comparison of Nigel [O I] $557.7 \mathrm{~nm}$ emission intensity (top; units are rayleighs) and TED total energy flux (bottom; units are ergs $\mathrm{cm}^{-2} \mathrm{~s}^{-1}$ ). Vertical dashed lines show a number of events common to both data sets, classified visually. See the electronic edition of the PASP for a color version of this figure. 


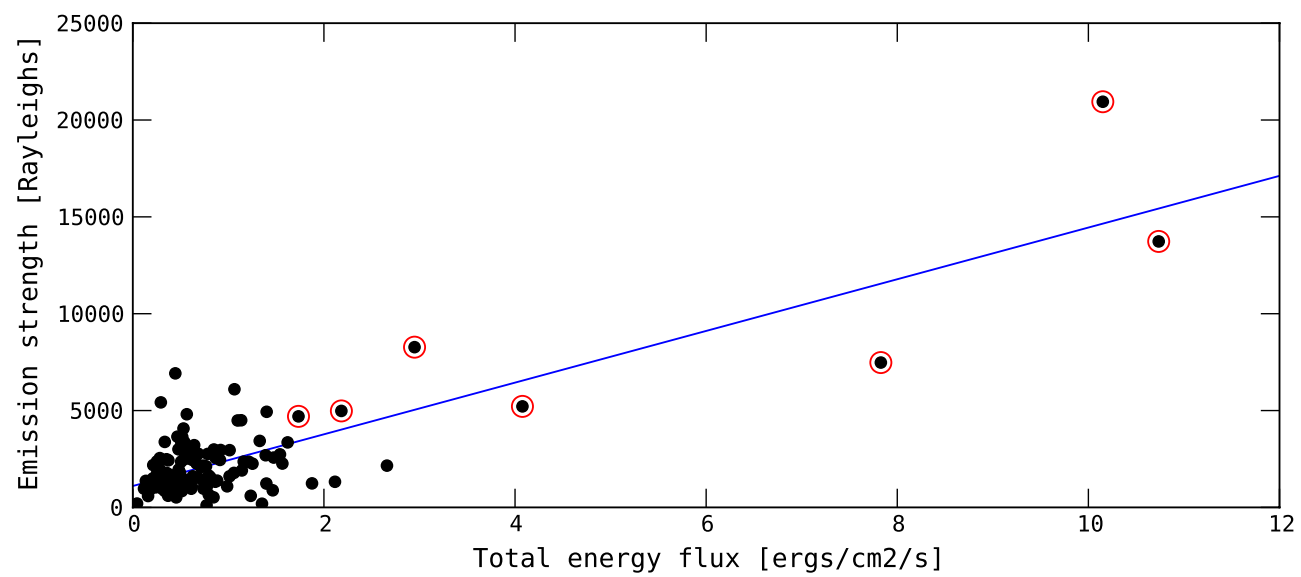

FIG. 13.-Correlation between Nigel [O I] $557.7 \mathrm{~nm}$ emission intensity and satellite total energy flux, using maximum values from 133 individual days. The identified auroral events from Fig. 12 have been marked with open circles, and the linear best-fit line $\left(R^{2}=0.64\right)$ is also shown. See the electronic edition of the $P A S P$ for a color version of this figure.

emissions. So we use a cutoff intensity of $750 \mathrm{R}$ for the [O I] $557.7 \mathrm{~nm}$ line, and we define observations below this intensity as low and those above it as high. The $750 \mathrm{R}$ limit is a reasonable estimate for the maximum expected value of the [O I] $557.7 \mathrm{~nm}$ emission in airglow (Patat 2008). It is likely that the low category consists largely of airglow, with some weak auroral contribution, and the high category is dominated by aurora.

Figures 9 and 10 show the variability of the low and high emissions, respectively. The time of magnetic midnight on the high-intensity plot was determined using the corrected geomagnetic coordinates and the IGRF/DGRF (international/definitive geomagnetic reference field) model-parameter calculator ${ }^{15}$ and was given to be $1 \mathrm{UTC}$, corresponding to $\sim 6 \mathrm{LT}$.

The low-intensity emissions, presumably consisting largely of airglow, with some weak auroral contribution, exhibit a strong diurnal pattern, with a minimum shortly after local midnight. Peak-to-peak fluctuations are around $400 \mathrm{R}$ in each case, with the exception of $\mathrm{N}_{2}^{+} 427.8 \mathrm{~nm}$, which shows a total variance of just $200 \mathrm{R}$. The high-intensity emissions, presumably largely for aurora, display very different behavior, most notable of which is a very strong increase prior to magnetic midnight at $4 \mathrm{LT}$ (10 MLT). The exception in this case is the [O I] $630.0 \mathrm{~nm}$ line, which shows no such increase, supporting the findings of Patat (2008) that the [O I] 557.7 and $630.0 \mathrm{~nm}$ features show only a very weak correlation.

Figure 11 shows the [O I] $557.7 \mathrm{~nm}$ variations in more detail, including error bars to indicate the spread of values in each bin. The number of observations and fraction of high-intensity emissions are also shown. The low number of observations around local midday is a natural consequence of the periods during which the Sun was close to, or above, the horizon. While we have previously stated that the strongest emissions occur prior

\footnotetext{
${ }^{15}$ See http://omniweb.gsfc.nasa.gov/vitmo/cgm_vitmo.html.
}

to magnetic midnight, the frequency of emissions greater than $750 \mathrm{R}$ is significantly higher from 10-23 LT (4-17 MLT), with two peaks around 15 and 20 LT (9 and 14 MLT).

\section{COMPARISON WITH SATELLITE MEASUREMENTS}

A fleet of polar-orbiting environmental satellites (POES) continuously monitors a variety of weather phenomena, including space weather and geomagnetic activity. The total-energy detector (TED), part of the upgraded space environment monitor (SEM-2), which is present on all POES platforms, is one such instrument that monitors the energy flux carried by auroral particles (electrons and protons) into the polar atmosphere. ${ }^{16}$

Given the relatively long integration times (512 s) and large field of view $\left(25^{\circ}\right)$ of Nigel observations, any detected auroral events will be undersampled in both space and time. With typical auroral evolution times of minutes, and overhead satellite passes just a few times per day, we consider a large area $\left( \pm 1.5^{\circ}\right.$ in latitude, $\pm 5.5^{\circ}$ in longitude) centered on Dome A for this study. The area was chosen to include approximately all the sky visible from Dome A up to a zenith distance of $60^{\circ}$ at $120 \mathrm{~km}$ altitude: the height at which the auroral particles detected by the satellite will enter the atmosphere. The geographic coordinates at this altitude are known as foot-of-field coordinates.

Data for every day in 2009 (in $16 \mathrm{~s}$ averages) from all six POES satellites (MetOp-02 and NOAA-15/16/17/18/19) were obtained from the POES SEM data access porta ${ }^{17}$ and filtered by foot-of-field coordinates to include the aforementioned area. The TED total energy flux is shown against the Nigel [O I] $557.7 \mathrm{~nm}$ emission intensities in Figure 12. As a means of

\footnotetext{
${ }^{16}$ From SEM-2 documentation; see http://satdat.ngdc.noaa.gov/sem/poes/ docs/sem2_docs/2006/SEM2v2.0.pdf.

${ }^{17}$ See http://www.ngdc.noaa.gov/stp/satellite/poes/dataaccess.html.
} 


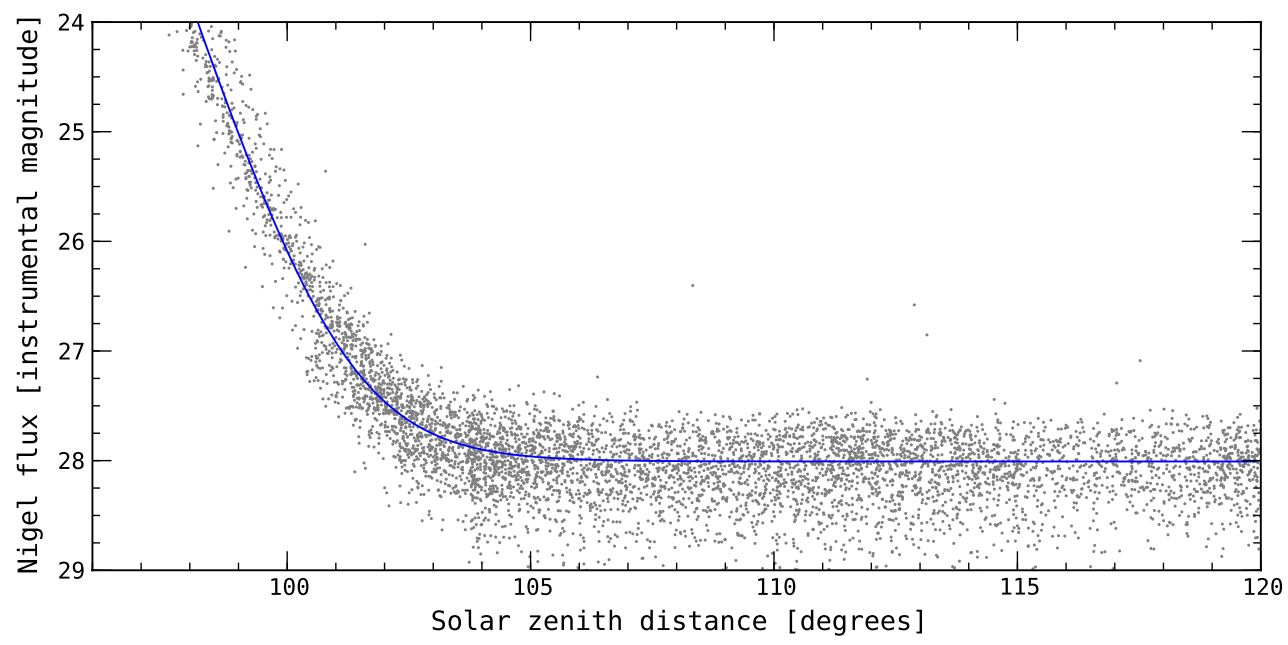

FIG. 14.-Sample calculation from pixel $400(\lambda=545.8 \mathrm{~nm})$ in the Nigel spectra. See the electronic edition of the PASP for a color version of this figure.

allowing for the temporal and spatial variations of both data sets, a corresponding correlation plot was produced using only the daily maximum values and is presented in Figure 13.

Reassuringly, a number of strong aurorae are detected by both Nigel and the POES fleet. Overall, there is a clear correlation $\left(R^{2}=0.64\right)$, particularly evident over the stronger events $(557.7 \mathrm{~nm}$ emission $>5 \mathrm{kR}$; total energy flux $>$ $2 \operatorname{ergs~} \mathrm{cm}^{-2} \mathrm{~s}^{-1}$ ). Note that we do not expect a strong correlation, since Nigel's FOV is quite different from that of the satellite, and there are also temporal offsets in the time of observations. While it may be possible to use a more elaborate approach to effectively increase the temporal and spatial resolution-such as the OVATION Prime model (Newell et al. 2010) or the auroral oval predictions produced by NOAA ${ }^{18}$ - it is beyond the scope of this article. If a strong correlation were found using high-resolution data, decades of archival satellite measurements could be used to study the likely historical fluctuations in auroral intensity as would have been observed from the ground.

\section{TWILIGHT AND THE AVAILABLE DARK TIME}

Midwinter periods at polar sites such as Dome A are characterized by long and continuous periods of uninterrupted darkness. There are also long periods of twilight in both the preceding and following months, however, due to the apparent near-horizontal motion of the Sun. The formal definition of astronomical night time is when the solar zenith distance, $\zeta_{\odot}$, reaches $108^{\circ}$, which, for some observing locations, suffices as an adequate description. For more exceptional sites, such as La Silla Paranal Observatory in the Atacama desert in Chile, a definition of $\zeta_{\odot}=105^{\circ}$ is more appropriate (Patat et al. 2006). At high latitudes, where the amount of usable dark time is

\footnotetext{
18 See http://www.swpc.noaa.gov/pmap/pmapS.html and http://helios .swpc.noaa.gov/ovation/South.html.
}

critically dependent on this value (e.g., Zou et al. 2010), it is important that this point is defined accurately. One interesting consequence of the low aerosol content of the atmosphere above the Antarctic plateau is that it contributes to the sky darkening faster than usual, as the intensity of multiply scattered light is minimized (Kenyon \& Storey 2006 and references therein).

We define the end of twilight as the solar zenith distance at which the median moonless dark sky brightens by $0.5 \mathrm{mag}-\mathrm{a}$ conservative estimate given typical variations of $0.1 \mathrm{mag}$ throughout a night (see $\S 2$ ). We denote the zenith distance of complete darkness $\zeta_{D}$. Using each wavelength interval from the Nigel spectra, averaged over 5 pixels $(\sim 2.5 \mathrm{~nm})$ to increase the $\mathrm{S} / \mathrm{N}$, we examined the instrumental magnitude as a function of $\zeta_{\odot}$ and fit an exponential curve to the data. The parameters of the fit allow us to determine at which solar zenith distance twilight ends using the definition above. We performed the same analysis for each Nigel observation multiplied and integrated with Bessell $U B V R I$ and SDSS ugriz filters, as well as the Gattini data for the $B V R$ bands.

Figure 14 shows the flux versus $\zeta_{\odot}$ for a single wavelength. A visual comparison with similar data and models presented in Patat et al. (2006) reveal that the sky does indeed darken faster at Dome A, supporting the idea that multiple scattering is less effective for sites on the Antarctic plateau. The amount of annual dark time and solar zenith distance at which twilight ends, $\zeta_{D}$, as a function of wavelength, is given in Figure 15. The average value of $\zeta_{D}$ over the wavelength interval was determined to be $102.6^{\circ}$. The amount of annual dark time was calculated to the nearest minute and ignores refraction effects and the contribution from moonlight. Small discrepancies exist between the Nigel and Gattini results, perhaps due to sensitivity differences.

The shape of the line resembles a twilight spectrum with numerous Fraunhofer absorption features. It is well established that $\mathrm{N}_{2}^{+}$emissions are enhanced during twilight (e.g., Slipher 1933; Bates 1949; Chamberlain \& Sagan 1960; Hunten 1963, 


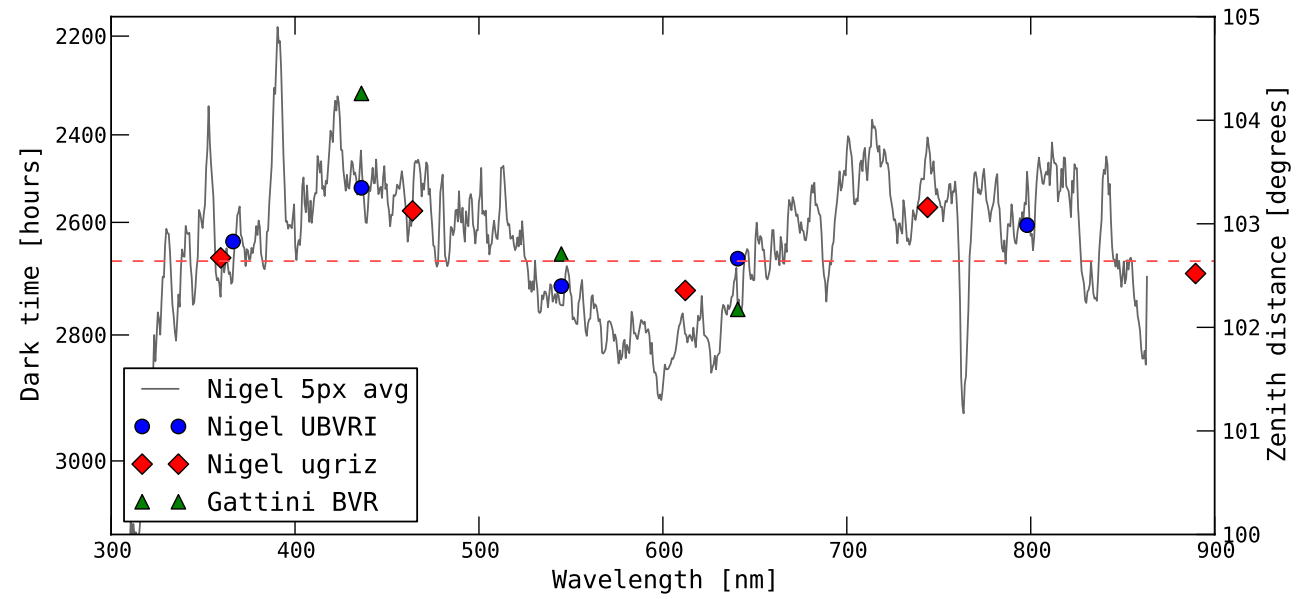

FIG. 15. - Solar zenith distance at which twilight ends, $\zeta_{D}$, as a function of wavelength and the $U B V R I$ and ugriz photometric bands (data points are plotted at their central wavelengths). The dashed horizontal line represents the average over the wavelength interval $300-850 \mathrm{~nm}$. The dips at 759 and $687 \mathrm{~nm}$ are the Fraunhofer $A$ and $B$ bands caused by terrestrial $\mathrm{O}_{2}$ absorption. See the electronic edition of the $P A S P$ for a color version of this figure.

1967), with stronger enhancements predicted in a magnetically disturbed atmosphere (a frequent occurrence at Dome A given the presence of nitrogen emissions in the median dark sky spectra shown previously in $\S 5$ ). A number of these enhancements are visible in the plot (e.g., $391.4 \mathrm{~nm}$ ) and cause a delayed onset of darkness, although positive identification of all lines is difficult as a result of "contamination" by Fraunhofer absorption lines.

Using the Paranal definition of $\zeta_{D}=105.0^{\circ}$, the amount of dark time at Dome A is $2161 \mathrm{hr}$ per year. Using a more appropriate definition of $\zeta_{D}=102.6^{\circ}$ we achieve, on average, $2670 \mathrm{hr}$ of dark time per year for observations in the $300-850 \mathrm{~nm}$ spectral range. Previously, simple visual analyses using data from the Antarctic plateau have reached similar conclusions (e.g., Moore et al. 2008; Sims et al. 2010; Zou et al. 2010), but as far as we are aware, this is the first quantitative result.

\section{CONCLUSION}

At Dome A, the dominant airglow and auroral emission lines have been examined using spectroscopic data from the Nigel experiment obtained close to solar minimum in 2009. The median contributions from the emissions are in good agreement with prior expectations based on models and are typically weaker than (and, at worst, equal to) the zodiacal light at a midlati- tude site. Our spectra reveal weak, near-constant $\mathrm{N}_{2}^{+}$emissions, which indicate that the polar atmosphere is always magnetically disturbed to some extent, although the exact mechanism for this disturbance is yet to be identified. The amount of dark time available at Dome A per year is found to be, on average, over $500 \mathrm{hr}$ more than what is expected using the definition for astronomical darkness at Paranal Observatory.

This research is supported by the Chinese PANDA International Polar Year project and the Polar Research Institute of China. The authors wish to thank all the members of the 2008/2009/2010 PRIC Dome A expeditions for their heroic efforts in reaching the site and for providing invaluable assistance to the expedition astronomers in setting up the PLATO observatory and its associated instrument suite. This research is financially supported by the Australian Research Council, the Australian Antarctic Division, the Chinese Academy of Sciences, the National Natural Science Foundation of China, the US National Science Foundation, and the United States Antarctic Program. Additional financial contributions have been made by the institutions involved in this collaboration. This research has made use of the SIMBAD database, operated at CDS, Strasbourg, France. We thank Gary Burns for helpful comments on the draft of this article.

\section{REFERENCES}

$\rightarrow$ Akasofu, S.-I. 2010, Eos, 91, 269

$\rightarrow$ Bates, D. R. 1949, Proc. R. Soc. London A, 196, 217

$\rightarrow$ Benn, C. R., \& Ellison, S. L. 1998, NewA Rev., 42, 503

$\rightarrow$ Bessell, M. S. 1979, PASP, 91, 589

$\rightarrow$ Bonner, C. S., et al. 2010, PASP, 122, 1122

$\rightarrow$ Brenton, J. G., \& Silverman, S. M. 1970, Planet. Space Sci., 18, 641

$\rightarrow$ Broadfoot, A. L., \& Kendall, K. R. 1968, J. Geophys. Res., 73, 426 Chamberlain, J. W. 1961, Physics of the Aurora and Airglow (New York: Academic Press) $\rightarrow$ Chamberlain, J. W., \& Sagan, C. 1960, Planet. Space Sci., 2, 157

$\rightarrow$ Dempsey, J. T., Storey, J. W. V., \& Phillips, A. 2005, PASA, 22, 91

$\rightarrow$ Deutsch, K. A., \& Hernandez, G. 2003, J. Geophys. Res., 108, 1430

$\rightarrow$ Feldstein, Y. I., \& Starkov, G. V. 1967, Planet. Space Sci., 15, 209

$\rightarrow$ Forsyth, R. J., \& Wraight, P. C. 1987, Planet. Space Sci., 35, 1449

$\rightarrow$ Fukuyama, K. 1976, J. Atmos. Terr. Phys., 38, 1279

Gattinger, R. L., \& Jones, A. V. 1974, Canadian J. Phys., 52, 2343

$\rightarrow$ Gussenhoven, M. S., \& Madden, D. 1990, J. Geophys. Res., 95, 10399 
$\rightarrow$ Hartz, T. R., \& Brice, N. M. 1967, Planet. Space Sci., 15, 301

$\rightarrow$ Holzworth, R. H., \& Meng, C.-I. 1975, Geophys. Res. Lett., 2, 377

$\rightarrow$ Hosokawa, K., Moen, J. I., Shiokawa, K., \& Otsuka, Y. 2011, J. Geophys. Res., 116, 1305

$\rightarrow$ Hunten, D. M. 1963, Planet. Space Sci., 10, 37 . 1967, Space Sci. Rev., 6, 493

$\rightarrow$ Jones, A. V., \& Gattinger, R. L. 1975, Canadian J. Phys., 53, 1806

$\rightarrow$ Kenyon, S. L., \& Storey, J. W. V. 2006, PASP, 118, 489

$\rightarrow$ Krisciunas, K. 1997, PASP, 109, 1181

$\rightarrow$ Kvifte, G. 1959, J. Atmos. Terr. Phys., 16, 252

$\rightarrow$ Lawrence, J. S., Ashley, M. C. B., Hengst, S., Luong-van, D. M., Storey, J. W. V., Yang, H., Zhou, X., \& Zhu, Z. 2009, Rev. Sci. Instrum., 80, 064501

$\rightarrow$ Li, Q., Xu, J., Yue, J., Yuan, W., \& Liu, X. 2011, Ann. Geophys., 29, 1401

$\rightarrow$ McEwen, D. J., \& Harrington, D. A. 1991, Canadian J. Phys., 69, 1055

$\rightarrow$ Meinel, A. B. 1950a, ApJ, 111, 555

$\rightarrow \longrightarrow$. 1950b, ApJ, 112, 120

$\rightarrow$ Meriwether, J. W., Jr., \& Walker, J. C. G. 1980, J. Geophys. Res., 85,1279

$\rightarrow$ Moen, J., Gulbrandsen, N., Lorentzen, D. A., \& Carlson, H. C. 2007, Geophys. Res. Lett., 34, 14113

$\rightarrow$ Moore, A., et al. 2008, Proc. SPIE, 7012, 701226

$\rightarrow$ Moore, A. M., et al. 2010, Proc. SPIE, 7733, 77331S

$\rightarrow$ Moore, J. G. 1963, J. Geophys. Res., 68, 1335

$\rightarrow$ Newell, P. T., Liou, K., \& Wilson, G. R. 2009, J. Atmos. Sol.-Terr. Phys., 71, 199

$\rightarrow$ Newell, P. T., Sotirelis, T., \& Wing, S. 2010, J. Geophys. Res., 115,3216 $\rightarrow$ Patat, F. 2008, A\&A, 481, 575

$\rightarrow$ Patat, F., Ugolnikov, O. S., \& Postylyakov, O. V. 2006, A\&A, 455, 385

$\rightarrow$ Pych, W. 2004, PASP, 116, 148

$\rightarrow$ Rayleigh, L. 1924, Proc. R. Soc. London A, 106, 117

$\rightarrow$ Sandford, B. P. 1961, Nature, 190, 245

$\rightarrow$. 1962, J. Atmos. Terr. Phys., 24, 155

$\rightarrow \longrightarrow$. 1964, J. Atmos. Terr. Phys., 26, 749

$\rightarrow$ Sandford, P. B. 1968, J. Atmos. Terr. Phys., 30, 1921

$\rightarrow$ Saunders, W., et al. 2009, PASP, 121, 976

$\rightarrow$ Shepherd, G. G., Roble, R. G., McLandress, C., \& Ward, W. E. 1997, J. Atmos. Sol.-Terr. Phys., 59, 655

Simmons, D. A. R. 1998, J. British Astron. Assoc., 108, 247

$\rightarrow$ Simmons, D. A. R., \& Henriksen, K. 1995, Polar Rec., 31, 315

$\rightarrow$ Sims, G., et al. 2010, Proc. SPIE, 7733, 77334M

$\rightarrow \longrightarrow$. 2012, PASP, 124, 74

Slipher, V. M. 1933, MNRAS, 93, 657

$\rightarrow$ van Dokkum, P. G. 2001, PASP, 113, 1420

$\rightarrow$ von Biel, H. A. 1990, J. Atmos. Terr. Phys., 52, 687

$\rightarrow$ Vorobjev, V. G., Kornilov, I. A., Kornilova, T. A., Yagodkina, O. I., Sandholt, P. E., \& Lybekk, B. 2008, Geomagn. Aeron., 48, 606

$\rightarrow$ Walker, M. F. 1988, PASP, 100, 496

$\rightarrow$ Winningham, J. D., \& Heikkila, W. J. 1974, J. Geophys. Res., 79, 949

$\rightarrow$ Yang, H., et al. 2009, PASP, 121, 174

$\rightarrow \longrightarrow$. 2010, PASP, 122, 490

Yntema, L. 1909, Publ. Kapteyn Astron. Lab. Groningen, 22, 1

$\rightarrow$ Zhu, L., Schunk, R. W., \& Sojka, J. J. 1997, J. Atmos. Sol.-Terr. Phys., 59, 1087

$\rightarrow$ Zou, H., et al. 2010, AJ, 140, 602 